\title{
初始制动时预测湿式制动器热对流特性的积分方法
}

\author{
赵 波 ${ }^{1,2}$ \\ (1. 四川大学机械工程学院 成都 610065 ; \\ 2. 先进制造技术四川省重点实验室 成都 610065)
}

\begin{abstract}
摘要: 湿式制动器对重型车辆安全具有关键影响, 以湿式制动器摩擦副间隙的冷却液压油(Automotive transmission fluid, ATF) 为研究对象, 考虑车辆初始制动阶段流体的黏性摩擦和层流流动特征, 利用积分方法建立了摩擦副流体的非稳态能量方程, 并获得了能同时满足轴向和径向边界条件的三维温度和热流密度显式解析表达式，通过简化动量方程和多项式分布假设也获 得了径向速度和压力的理论解, 与以往试验对比表明, 压力和温度解析解与试验结果具有较好的一致性, 理论模型有望推广 用于液黏离合器等其他 HVD 装置的 ATF 速度场、温度场和热流密度场的理论预测。
\end{abstract}

关键词：湿式制动器；液体㯟性传动；积分法；温度预测；热流密度；初始制动；层流流动

中图分类号: TH243; U461

\section{Integration Method of Thermal Convection Characteristics Prediction for Unsteady Laminar Flows during Initial Braking Stage of Wet Brakes}

\author{
ZHAO Bo ${ }^{1,2}$ \\ (1. School of Mechanical Engineering, Sichuan University, Chengdu 610065; \\ 2. Sichuan Provincial Key Lab of Advanced Manufacturing Technology, Chengdu 610065)
}

\begin{abstract}
A wet brake has a crucial influence on the safety of heavy-duty vehicles. A theoretical thermal analysis is conducted on the automotive transmission fluid (ATF) within a wet brake for initial braking stage. The energy equation of ATF is formulated using the integration method for unsteady laminar flows. An analytical model assuming the polynomial distribution pattern is developed to approximate the temperature rise due to the hydroviscous friction and relative motion between the separator disks and friction disks of the wet brake. The closed-form analytical solutions of three-dimensional (3D) temperature and heat fluxes of convective heat transfer are obtained, respectively, which can satisfy all the boundary conditions for both radial and axial directions. The theoretical expressions of the radial velocity and pressure of ATF are also proposed based on the simplified Navier-Stokes equation. Finally, the temperature rises and pressure predicted by the analytical models are validated by comparing with the previous experimental data. The analytical methods of 3D velocity, temperature and heat flux proposed might have the potentials to be extended to predict those of HVD (hydroviscous drive) devices such as hydroviscous clutches, dynamometers, and so on.
\end{abstract}

Key words: wet brake; hydroviscous drive; integration method; thermal analysis; heat flux; initial braking; laminar flow

\section{0 前言}

车辆制动过程是一个能量转换过程, 车辆的机 械能通过制动器和轮胎的摩擦转换为热能并及时散 发掉, 能量转化的核心部件是制动器, 其制动特性、 可靠性和使用寿命对车辆安全具有关键影响, 正是 由于制动器摩擦副间产生摩擦力才实现制动并产生

20191205 收到初稿, 20200507 收到修改稿
热量的, 见图 1。与干盘式制动器相比, 湿式多盘 制动器具有制动转矩大、制动性能稳定、散热效果 好、抗热衰退与抗污染能力强、寿命长和免于维护 等优点 ${ }^{[1-6]}$, 常被采用为矿用汽车、农业机械、军用 轮式车辆和坦克等重型装备的制动装置。

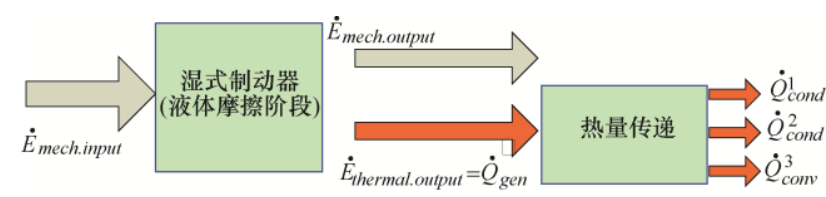

图 1 湿式制动器在液体摩擦阶段的能量流 
湿式多盘制动器常采用密闭式腔体, 内置多片 旋转的摩擦盘与滚动轮转相连接, 对应多片非旋转 的对偶盘与固定桥壳相连接, 二者之间充满了持续 流动的冷却液压油(Automotive transmission fluid, ATF), 见图 2。在活塞压力作用下, 初始制动阶段 中的摩擦副利用液体黏性传动(Hydroviscous drive, HVD) 产生制动转矩 ${ }^{[7-8]}$, ATF 承担着制动和散热的 双重功能, 该阶段称液体摩擦阶段。在活塞力持续 作用下，持续制动阶段中的摩擦盘和对偶盘轴向 间隙趋于零, 摩擦副表面开始贴合, 此时主要靠 机械摩擦力形成制动转矩, 直至将摩擦盘的旋转 角速度降至零, 同时将传递进来的机械能全部转 化为热能。

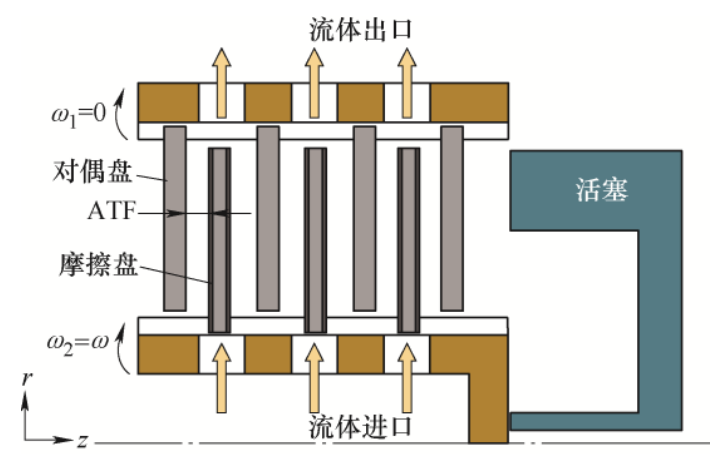

图 2 湿式多盘制动器结构示意图

从时间维度上，通常将整个制动过程划为三个 阶段 ${ }^{[9-10]}$ : 初始制动的液体摩擦阶段、持续制动的 机械摩擦阶段和制动结束后的非作业冷却阶段, 如 图 3 所示。在液体摩擦阶段制动转矩主要由液体黏 性产生的液体摩擦力提供, 这个阶段经历时间非常 短(零点几秒到一秒), 摩擦副传热主要通过非稳态 流体的热对流实现; 在机械摩擦阶段制动转矩主要 由摩擦盘和对偶盘表面微凸体接触产生机械摩擦力 提供, 持续时间相对较长(几秒至十几秒), 传热主 要靠摩擦副的稳态热传导方式 ${ }^{[11]}$ 。在初始制动阶段, 随着车辆制动强度迅速加大, 在极短时间内摩擦副 表面产生大量热量并导致摩擦副温度急剧升高, 若 温升超过一定限度, 将引起摩擦祄层的制动性能衰 退、摩擦副表面产生裂纹和局部破坏、热弹性失稳、 过早磨损等, 导致制动失效 ${ }^{[12-16]}$ 。为此, 制动过程 中采用 ATF 持续对摩擦盘、对偶盘散热冷却, 以保 证湿式制动器的正常作业和工作寿命。在满足车辆 制动效能强制标准的前提下, 湿式制动器设计前需 要准确预测制动器的温度场和热流密度场特性, 尤 其是作业时间极短的初始制动阶段的对流传递特 性, 为后续制动器的传矩、传热等计算提供设计基 础 $^{[5,13-14]}$ 。

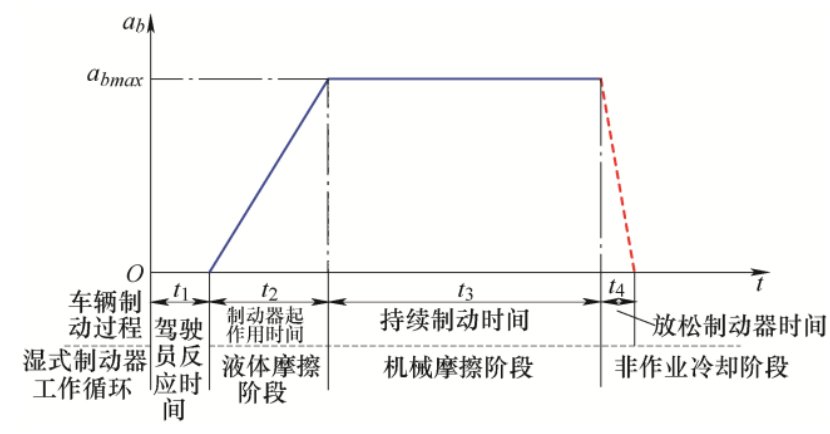

图 3 车辆整个制动过程的阶段组成 $\left(a_{b}\right.$ 为制动减速度)

湿式制动器作业过程中, 具有液体黏性摩擦机械接触摩擦-生热/传热/散热等高度交织的热、机、 流、固耦合等特征, 使得温度场和传热特性的研究 一直存在较大挑战。目前对湿式制动器的温度场和 传热特性研究多采用数值分析方法, 获得湿式制动 器 ATF 非稳态温度场二维显式解析解的研究还鲜有 报道。本文的主要内容如下: 在前期带排扭矩作用 下湿式制动器温度场理论预测等工作基础上 ${ }^{[17]}$, 考 虑初始制动时液体黏性摩擦特征, 拟采用多项式分 布模式假设, 得到能同时满足轴向和径向边界条件 的瞬态速度场、温度场和热流密度场的三维解析模 型, 然后利用积分方法建立湿式制动器 ATF 的非稳 态能量方程, 最后获得相应的显式解析解。

\section{1 理论假设}

采用理论假设如下: (1) ATF 流速相对较低且完 全充满摩擦盘和对偶盘中间, 视为层流流动, ATF 沿周向为 Couette 流动, 摩擦副和 ATF 沿周向速度

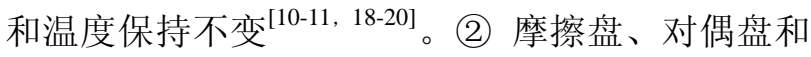
ATF 是匀质、各向同性材料, ATF 为黏性不可压缩 流体, 摩擦盘和对偶盘表面无滑移; 在温升不太大 的情况下, 视 ATF 的动力粘度 $\mu$ 、运动粘度 $v$ 、比热 容 $c$ 和热传导率 $\lambda$ 不随温度 $T$ 变化。(3) 摩擦盘祄片 的比热容和热导率均相对较低, 视摩擦盘为热绝缘 体, 不考虑油槽影响; 液体摩擦阶段持续时间非常 短, 对偶盘尚来不及进行传热和散热, 初始制动阶 段的传热只由 ATF 承担 ${ }^{[5,21]}$ 。(4) 摩擦盘和对偶板 始终保持对中无偏斜状态, 忽略惯性力和体积力影 响 ${ }^{[14]}$ 。(5) 忽略各摩擦副轴向速度的差异, 认为每 个摩擦副的速度场分布和温度场分布均相同。(6) 通 常热辐射传热比例不足 5\% 10\%，忽略不计。

\section{2 液体摩擦阶段速度场的理论分布}

预测 ATF 温度场之前, 需要先获得 ATF 速度场 
的分布规律。以湿式制动器中充满摩擦盘、对偶盘 间隙的流体 ATF 为研究对象, 建立图 4 所示柱坐标 系, 其中 $r_{1} \leqslant r \leqslant r_{2}, 0 \leqslant \theta \leqslant 2 \pi, 0 \leqslant z \leqslant h, r 、 \theta$ 、 $z$ 分别为柱坐标系的径向、周向和轴向坐标, $r_{1}$ 和 $r_{2}$ 分别代表摩擦副和 ATF 的内径和外径。与车轮同 轴的摩擦盘以角速度 $\omega_{2}$ 旋转 $\left(\omega_{2}=\omega\right)$, 它由中间芯层 和两侧粘接的摩擦衬层组成; 对偶盘转动角速度 $\omega_{1}=0$, 摩擦盘和对偶盘在活塞压力 $p$ 作用下可沿轴 向 $z$ 自由平移; 在摩擦盘和对偶盘之间充满了 ATF, 油膜厚度为 $h$ 。

针对 ATF, 进一步简化为 ${ }^{[22]}$ : (1) 流体轴向 速度 $v_{z}$ 远小于径向速度 $v_{r}$ 和周向速度 $v_{\theta}$, 即 $v_{z}=0$; (2) 除压力 $p$ 外, 其他任何变量在轴向变化率远大于 径向和周向变化率; (3) 压力 $p$ 只与径向位移 $r$ 有关, 即 $\partial p / \partial z=\partial p / \partial \theta=0$ 。(4) $v_{r} 、 v_{\theta}$ 和温度 $T$ 沿周向变化为 零; 液体摩擦阶段制动时间非常短 $\left(\sim 10^{-2} \mathrm{~s}\right)$, 视 $v_{r}$ 、 $v_{\theta}$ 和对偶盘表面温度 $T_{w}$ 与时间变量 $t$ 无关 ${ }^{[23]}$ 。基于 此, 纳维-斯托克斯方程简化如下

$$
\left\{\begin{array}{l}
v_{r} \frac{\partial v_{r}}{\partial r}+v_{z} \frac{\partial v_{r}}{\partial z}-\frac{v_{\theta}^{2}}{r}=-\frac{1}{\rho} \frac{\partial p}{\partial r}+v \frac{\partial^{2} v_{r}}{\partial z^{2}} \\
v_{r} \frac{\partial v_{\theta}}{\partial r}+v_{z} \frac{\partial v_{\theta}}{\partial z}+\frac{v_{r} v_{\theta}}{r}=v \frac{\partial^{2} v_{\theta}}{\partial z^{2}} \\
\frac{\partial p}{\partial z}=0
\end{array}\right.
$$

\section{1 轴向速度}

根据 Soo 的理论简化 ${ }^{[22]}$, ATF 的轴向速度 $v_{z}=0$ 。

\section{2 周向速度}

油膜厚度 $h$ 非常薄 $(0.1 \sim 100 \mu \mathrm{m})$, 由理论假设 (1)和(2), 考虑到液压油为黏性流体, 对偶盘与流体 接触表面 $z=0$ 处, $v_{\theta}=0$; 摩擦盘与流体接触表面 $z=h$ 处, $v_{\theta}=\omega r$, 如图 4 所示, 根据 Soo 的推导 ${ }^{[22]}$ 和文 献[23], 液体摩擦阶段 ATF 周向速度 $v_{\theta}$ 沿轴向 $z$ 的 分布为

$$
v_{\theta}(r, z, t)=\omega r z / h
$$

注意到式(2)自动满足式(1)第二式。

根据文献[23], 因液体黏性摩擦(HVD)而产生的 热量大小, 只需考虑沿轴向变化的 ATF 周向速度 $v_{\theta}$, 其他方向的速度梯度对耗散热的贡献可忽略不计, 则单位体积产生的热流量, 即能量耗散函数 $\Phi\left(\mathrm{W} / \mathrm{m}^{3}\right)$ 为

$$
\Phi=\mu\left(\frac{\mathrm{d} v_{\theta}}{\mathrm{d} z}\right)^{2}
$$

将式(2)代入，得

$$
\Phi=\mu \omega^{2} r^{2} / h^{2}
$$

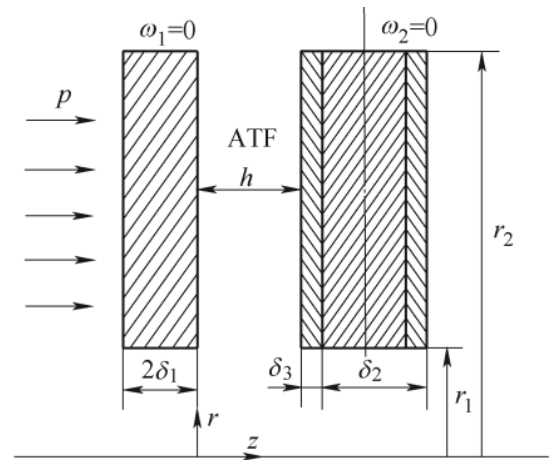

(a) 左侧为对偶盘, 右侧为摩擦盘

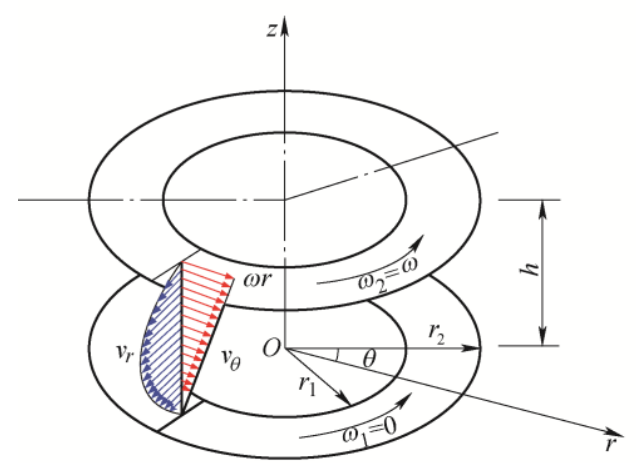

(b)下侧为对偶盘, 上侧为摩擦盘

图 4 湿式制动器内 ATF 的周向速度分布

则每个摩擦副表面产生的总热流量 $\dot{Q}_{g e n}$ 为

$$
\dot{Q}_{g e n}=\int_{r_{1}}^{r_{2}} \Phi 2 \pi r h \mathrm{~d} r=0.5 \mu \pi \omega^{2}\left(r_{2}^{4}-r_{1}^{4}\right) / h
$$

\section{3 径向速度}

设径向速度 $v_{r}$ 沿轴向 $z$ 的分布形式为

$$
v_{r}=a_{0}+a_{1} z+a_{2} z^{2}+a_{3} z^{3}
$$

式中, $a_{i}(i=0,1,2,3)$ 为待定系数。

如图 4 所示, 由理论假设(2)和(4), 摩擦盘和对 偶板始终保持对中且壁面流体无滑移, ATF 在摩擦 盘和对偶盘之间流动时，与摩擦盘和对偶盘表面接 触侧的径向速度分别等于摩擦盘/对偶盘的径向速 度 ${ }^{[3]}$, 即 $v_{r}=v_{r 1}=v_{r 2}=0$, 即

$$
\left.v_{r}\right|_{z=0}=0,\left.\quad v_{r}\right|_{z=h}=0
$$

考虑式(5)以及 $v_{z}=0$, 由动量方程式(1)中第一式, 在 ATF 与摩擦盘、对偶盘的边界表面处, 有

$$
\left.\frac{\partial^{2} v_{r}}{\partial z^{2}}\right|_{z=0, h}=\frac{1}{\mu} \frac{\partial p}{\partial r}-\frac{1}{v r}\left(\left.v_{\theta}\right|_{z=0, h}\right)^{2}
$$

将周向速度 $v_{\theta}$ 表达式(2)代入，整理得

$$
\left.\frac{\partial^{2} v_{r}}{\partial z^{2}}\right|_{z=0}=\frac{1}{\mu} \frac{\partial p}{\partial r},\left.\frac{\partial^{2} v_{r}}{\partial z^{2}}\right|_{z=h}=\frac{1}{\mu} \frac{\partial p}{\partial r}-\frac{\rho r \omega^{2}}{\mu}
$$

将径向速度 $v_{r}$ 的幂函数表达式代入式(5)、(6), 确定 
待定系数后得径向速度 $v_{r}$ 表达式为

$$
v_{r}=\frac{\rho r \omega^{2} h^{2}}{6 \mu}\left(\frac{z}{h}-\frac{z^{3}}{h^{3}}\right)-\frac{h^{2}}{2 \mu} \frac{\mathrm{d} p}{\mathrm{~d} r}\left(\frac{z}{h}-\frac{z^{2}}{h^{2}}\right)
$$

由质量守恒定律, ATF 的质量流量 $\dot{m}(\mathrm{~kg} / \mathrm{s})$ 可 定义为

$$
\dot{m}=\rho Q=\int_{0}^{h} \rho v_{r} \cdot 2 \pi r \mathrm{~d} z
$$

这里 $Q$ 指体积流量 $\left(\mathrm{m}^{3} / \mathrm{s}\right)$, 将式(7)代入式(8), 得

$$
\dot{m}=\frac{\rho \pi r h^{3}}{6 \mu}\left(\frac{\rho r \omega^{2}}{2}-\frac{\mathrm{d} p}{\mathrm{~d} r}\right)
$$

或者

$$
\frac{\mathrm{d} p}{\mathrm{~d} r}=\frac{\rho r \omega^{2}}{2}-\frac{6 \mu \dot{m}}{\rho \pi r h^{3}}
$$

式(9)两边沿 $r$ 积分, 考虑边界条件 $\left.p\right|_{r=r_{1}}=p_{0}$, 这里 $p_{0}$ 为 ATF 入口处压力, 得 ATF 的压力 $p$ 为

$$
p=p_{0}+\frac{\rho \omega^{2}}{4}\left(r^{2}-r_{1}^{2}\right)+\frac{6 \mu \dot{m}}{\rho \pi h^{3}} \ln \left(\frac{r_{1}}{r}\right)
$$

将式(9)代回式(7), 即得 $v_{r}$ 的最终表达式

$$
v_{r}=\frac{3 \dot{m}}{\rho \pi r h}\left(\frac{z}{h}-\frac{z^{2}}{h^{2}}\right)-\frac{\rho r \omega^{2} h^{2}}{12 \mu}\left(\frac{z}{h}-3 \frac{z^{2}}{h^{2}}+2 \frac{z^{3}}{h^{3}}\right)
$$

\section{ATF 热流密度场和能量积分方程}

圆柱坐标系中 $r 、 \theta 、 z$ 方向的热流密度分别记 为 $q_{r} 、 q_{\theta} 、 q_{z}$, 在比热容为常数时可写为

$$
\left\{\begin{array}{l}
q_{r}=-\lambda \frac{\partial T}{\partial r}+\rho c v_{r}\left(T-T_{0}\right) \\
q_{\theta}=-\frac{\lambda}{r} \frac{\partial T}{\partial \theta}+\rho c v_{\theta}\left(T-T_{0}\right) \\
q_{z}=-\lambda \frac{\partial T}{\partial z}+\rho c v_{z}\left(T-T_{0}\right)
\end{array}\right.
$$

式中, $T_{0}$ 为流体初始温度, 也是 ATF 入口温度, $c$ 是定压比热容 $[\mathrm{J} /(\mathrm{kg} \cdot \mathrm{K})], \rho$ 为流体密度 $\left(\mathrm{kg} / \mathrm{m}^{3}\right)$ 。注 意到所有速度变为零时 (热对流贡献为零), 式(12) 自动退化为描述热传导的傅立叶定律。

由能量平衡的微元分析易知

$$
\rho c \frac{\partial T}{\partial t}+\frac{\partial q_{r}}{\partial r}+\frac{q_{r}}{r}+\frac{1}{r} \frac{\partial q_{\theta}}{\partial \theta}+\frac{\partial q_{z}}{\partial z}=\Phi
$$

将式(11)、(3)代入式(12)就得到非稳态流动的对流能 量微分方程(包括热传导和热对流)。

在液体摩擦阶段, 由数量级分析易知, 湿式制 动器中 ATF 径向的热传导的贡献与热对流相比可忽 略; 而不计 ATF 轴向速度后轴向则只有热传导起作
用; 由理论假设 (1), 忽略周向温差后热传导贡献 为零, 则 ATF 周向仅存在因流体运动携带的温差 $\left(T-T_{0}\right)$ 导致的热对流。因此, 三个方向的热流密度 式(12)可进一步简化为

$q_{r}=\rho c v_{r}\left(T-T_{0}\right), \quad q_{\theta}=\rho c v_{\theta}\left(T-T_{0}\right), q_{z}=-\lambda \frac{\partial T}{\partial z}$

代入能量方程式(13)并考虑连续性方程和能量耗散 函数表达式(3), 得

$$
\rho c\left(\frac{\partial T}{\partial t}+v_{r} \frac{\partial T}{\partial r}\right)=\lambda \frac{\partial^{2} T}{\partial z^{2}}+\frac{\mu \omega^{2} r^{2}}{h^{2}}
$$

上述方程是二阶偏微分非齐次方程, 难于获得 显式解析解。为此, 拟采用能量积分的方法解决。 设在流场中划出几个不动的控制表面围成一个固定 的控制体，如图 5 所示。其控制表面如下：相距微 元距离 $\mathrm{d} r$ 的两个垂直于摩擦盘和对偶盘壁面的圆 柱面 $a b$ 和 $c d$; 摩擦盘表面圆环 $b d$ 和对偶盘表面圆 环 $a c$, 二者沿周向均匀分布, 轴向相距为 $h$ 。推导 时 ATF 径向的传导与对流相比可忽略不计, 只考虑 $\mathrm{ATF}$ 的径向流速 $v_{r}$, 忽略轴向速度 $v_{z}$, 由理论假设 (3), 周向速度产生的黏性耗散热 $\dot{Q}_{g e n}$ 只由 ATF 的对 流热传递项 $\dot{Q}_{\text {conv }}^{3}$ 带走, 忽略摩擦盘和对偶盘的传导 项 $\dot{Q}_{\text {cond }}^{1}$ 和 $\dot{Q}_{\text {cond }}^{2}$, 如图 5 所示。

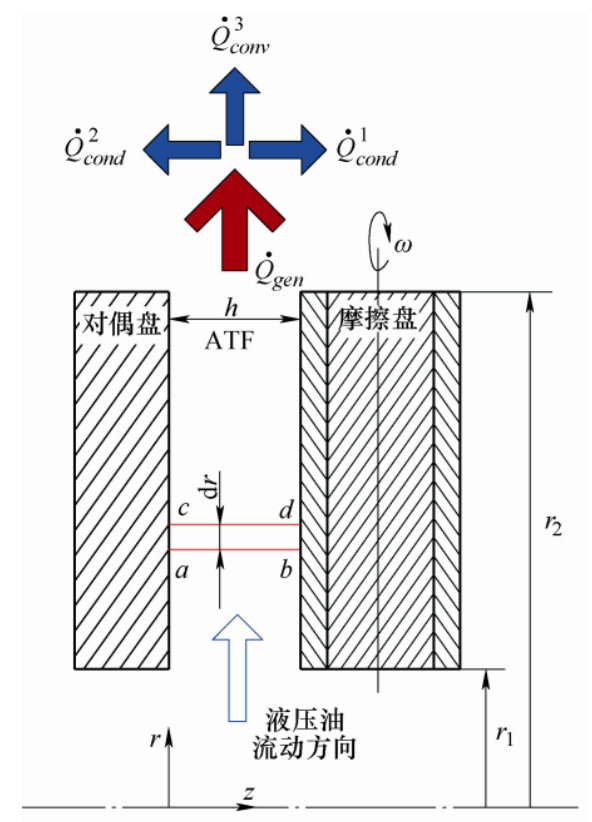

图 5 能量积分方程的推导

(1) 径向: 忽略 ATF 径向传导项, 由式(14)可 知单位时间通过控制面 $a b$ 进入控制体的热量为

$$
\int_{0}^{h} \rho c v_{r}\left(T-T_{0}\right) \cdot 2 \pi r \mathrm{~d} z
$$

同一时间间隔内, 通过控制面 $c d$ 流出控制体的热量为 
$\int_{0}^{h} \rho c v_{r}\left(T-T_{0}\right) \cdot 2 \pi r \mathrm{~d} z+\frac{\mathrm{d}}{\mathrm{d} r}\left(\int_{0}^{h} \rho c v_{r}\left(T-T_{0}\right) \cdot 2 \pi r \mathrm{~d} z\right) \mathrm{d} r$

(2) 轴向: 假定 ATF 在摩擦盘和对偶盘界面处 $b d$ 和 $a c$ 表面无热传导; 因 $v_{z}=0$, 故而对流项为零。

(3) 周向: 轴对称结构周向温度处处相等, 无 热传导; 对流产生的热流密度处处相等, 见式(14) 第二式。但是, 由微元分析易知, 沿周向净传递的 热流量为零。

(4) 由牛顿内摩擦定律, 液体存在黏性, 周向 速度 $v_{\theta}$ 沿轴向的梯度作用下会产生液体摩擦转矩, 同时机械能转化为热能。单位时间内单位体积生成 的热量大小为 $\Phi=\mu\left(\frac{\mathrm{d} v_{\theta}}{\mathrm{d} z}\right)^{2}$, 考虑式(3), 则在该控 制体内单位时间内生成的热量为

$$
\int_{0}^{h} \Phi 2 \pi r \mathrm{~d} r \mathrm{~d} z=\int_{0}^{h} \frac{2 \pi \mu \omega^{2} r^{3}}{h^{2}} \mathrm{~d} r \mathrm{~d} z=\frac{2 \pi \mu \omega^{2} r^{3}}{h} \mathrm{~d} r
$$

(5) 制动时是非稳态传热过程, 控制体 $a b d c$ 中 热力学能随时间的变化率为

$$
\int_{0}^{h} \rho c \frac{\partial T}{\partial t} \cdot 2 \pi r \mathrm{~d} r \mathrm{~d} z
$$

由能量平衡关系, 单位时间内净流入的热量加 上生成的热量等于控制体内热力学能的变化量, 即

$$
\begin{aligned}
& -\frac{\mathrm{d}}{\mathrm{d} r}\left(\int_{0}^{h} \rho c v_{r}\left(T-T_{0}\right) \cdot 2 \pi r \mathrm{~d} z\right) \mathrm{d} r+\frac{2 \pi \mu \omega^{2} r^{3}}{h} \mathrm{~d} r= \\
& \int_{0}^{h} \rho c \frac{\partial T}{\partial t} \cdot 2 \pi r \mathrm{~d} r \mathrm{~d} z
\end{aligned}
$$

在 $\rho c$ 为常数时, 式(16)进一步简化为

$$
\frac{\mu \omega^{2} r^{2}}{\rho c h}=\frac{1}{r} \frac{\mathrm{d}}{\mathrm{d} r}\left(\int_{0}^{h} v_{r}\left(T-T_{0}\right) r \mathrm{~d} z\right)+\int_{0}^{h} \frac{\partial T}{\partial t} \mathrm{~d} z
$$

这就是通过积分方法获得的能量方程, 它是能量微 分方程式(15)的近似等效式, 从而避免了数学上求 解偏微分方程的困难。

\section{4 液体摩擦制动阶段温度场理论分布}

令 $T_{w}$ 代表对偶盘的壁面温度, 引入过余温度, 即温升 $\Theta=T-T_{0}$, 并记 $\Theta_{w}=T_{w}-T_{0}, l=r-r_{1}$, $l_{0}=r_{2}-r_{1}$, 考虑热绝缘假设(3), 则 ATF 温升函数 $\Theta$ 沿轴向和径向的边界条件可写成 ${ }^{[7]}$

$$
\left.\Theta\right|_{z=0}=\Theta_{w},\left.\frac{\partial \Theta}{\partial z}\right|_{z=0, h}=0,\left.\Theta\right|_{l=0}=0,\left.\frac{\partial \Theta}{\partial l}\right|_{l=l_{0}}=0
$$

如前所述, 初始制动时间非常短, 有 $\partial \Theta_{w} / \partial t=0$, 考虑上面最后一个边界条件和式(5), 由能量微分方
程式(15)可获得新边界条件为: $\left.\frac{\partial^{2} \Theta}{\partial z^{2}}\right|_{l=l_{0}} ^{z=0}=-\frac{\mu \omega^{2} r_{2}^{2}}{\lambda h^{2}}$ 。

设 $\Theta$ 具有多项式的分布形式: $\Theta=b_{0}+\left(b_{1} z+\right.$ $\left.b_{2} z^{2}+b_{3} z^{3}\right)\left(c_{0}+c_{1} l+c_{2} l^{2}\right), b_{i}(i=0,1,2,3)$ 和 $c_{j}(j=0,1,2)$ 为待定系数, 由以上六个边界条件可确定这些待定 系数为

$b_{0}=\Theta_{w}, b_{1}=0, b_{2}=\frac{\mu}{2 \lambda} \frac{\omega^{2} r_{2}^{2}}{h^{2} l_{0}^{2} c_{2}}, b_{3}=-\frac{\mu}{3 \lambda} \frac{\omega^{2} r_{2}^{2}}{h^{3} l_{0}^{2} c_{2}}, c_{0}=0, c_{1}=-2 l_{0} c_{2}$

则 ATF 温度场分布的表达式确定为

$$
\Theta=\Theta_{w}+\frac{\mu \omega^{2}}{\lambda}\left(\frac{z^{2}}{2 h^{2}}-\frac{z^{3}}{3 h^{3}}\right)\left[\frac{\left(r-r_{1}\right)^{2}-2\left(r-r_{1}\right)\left(r_{2}-r_{1}\right)}{\left(1-r_{1} / r_{2}\right)^{2}}\right]
$$

在以上表达式中, $\Theta_{w}$ 尚未确定, 需利用能量积分方 程最终获得。重写方程式(17)为

$$
\frac{\mu \omega^{2} r^{2}}{\rho c h}=\frac{1}{r} \frac{\mathrm{d}}{\mathrm{d} r}\left(\int_{0}^{h} v_{r} \Theta r \mathrm{~d} z\right)+\int_{0}^{h} \frac{\partial \Theta}{\partial t} \mathrm{~d} z
$$

首先计算积分方程式(19)右侧第一项。分别将 径向速度表达式(10)和温升表达式(18)代入，有

$$
\begin{aligned}
& \frac{1}{r} \frac{\mathrm{d}}{\mathrm{d} r}\left(\int_{0}^{h} v_{r} \Theta r \mathrm{~d} z\right)=\frac{\dot{m}}{2 \rho \pi r} \frac{\partial \Theta_{w}}{\partial r}+\frac{\mu \dot{m} \omega^{2}\left(1-r_{2} / r\right)}{12 \rho \pi \lambda\left(1-r_{1} / r_{2}\right)^{2}}+ \\
& \frac{\rho h^{3} \omega^{4}}{1680 \lambda\left(1-r_{1} / r_{2}\right)^{2}}\left(2 r^{2}-3 r_{2} r-r_{1}^{2}+2 r_{1} r_{2}\right)
\end{aligned}
$$

为计算方程式(19)右侧第二项, 有必要先获得 $\mathrm{d} \omega / \mathrm{d} t$ 的表达式。在液体摩擦区，考虑 ATF 的 HVD 特征, 如图 4 所示, 摩擦盘和对偶盘间的油膜因存 在周向速度沿轴向的梯度, 根据牛顿内摩擦定律, 油膜切应力 $\tau$ 大小为

$$
\tau=-\mu \frac{\partial v_{\theta}}{\partial z}
$$

式中, $\mu$ 为液压油的动力粘度, $v_{\theta}$ 为 ATF 的周向速 度。因此, 具有 $N$ 个摩擦副的湿式制动器产生的总 制动转矩 $M$ 为 ${ }^{[2]}$

$$
M=N \int_{r_{1}}^{r_{2}} \tau 2 \pi r^{2} \mathrm{~d} r=-2 N \mu \pi \int_{r_{1}}^{r_{2}} \frac{\partial v_{\theta}}{\partial z} r^{2} \mathrm{~d} r
$$

将式(2)代入式(21), 积分得

$$
M=-\frac{N \mu \pi \omega}{2 h}\left(r_{2}^{4}-r_{1}^{4}\right)
$$

设 $m_{V}$ 代表双桥汽车总质量, $R$ 代表车轮滚动 半径, 前、后桥各安装 2 个湿式制动器, 考虑车 辆下坡时载荷的转移效应, 设前、后桥制动力分 配比例固定为 $2: 3$ (也可取其他值, 计算方法相 同), 安装在后桥的单台湿式制动器制动转矩 $M=3 / 10 m_{V} R^{2} \mathrm{~d} \omega / \mathrm{d} t$, 与式(21)联立得 


$$
\frac{\mathrm{d} \omega}{\mathrm{d} t}=-\frac{5 N \mu \pi \omega}{3 m_{V} R^{2} h}\left(r_{2}^{4}-r_{1}^{4}\right)
$$

然后计算式(17)右侧第二项。将温升表达式(16) 代入，考虑 $\partial \Theta_{w} / \partial t=0$, 整理得

$$
\int_{0}^{h} \frac{\partial \Theta}{\partial t} \mathrm{~d} z=\frac{\mu h \omega}{6 \lambda} \frac{\mathrm{d} \omega}{\mathrm{d} t}\left[\frac{\left(r-r_{1}\right)^{2}-2\left(r-r_{1}\right)\left(r_{2}-r_{1}\right)}{\left(1-r_{1} / r_{2}\right)^{2}}\right]
$$

将前面的 $\mathrm{d} \omega / \mathrm{d} t$ 表达式代入

$$
\int_{0}^{h} \frac{\partial \Theta}{\partial t} \mathrm{~d} z=\frac{5 N \pi \mu^{2} \omega^{2}\left(r_{2}^{4}-r_{1}^{4}\right)}{18 m_{v} R^{2} \lambda}\left[\frac{2\left(r-r_{1}\right)\left(r_{2}-r_{1}\right)-\left(r-r_{1}\right)^{2}}{\left(1-r_{1} / r_{2}\right)^{2}}\right]
$$

将式(22)和式(18)代入非稳态能量积分方程式(19), 得

$$
\begin{aligned}
\frac{\mathrm{d} \Theta_{w}}{\mathrm{~d} r}= & \frac{2 \pi \mu \omega^{2} r^{3}}{c \dot{m} h}+\frac{\mu \omega^{2}\left(r_{2}-r\right)}{6 \lambda\left(1-r_{1} / r_{2}\right)^{2}}-\frac{\pi \rho^{2} h^{3} \omega^{4}}{840 \dot{m} \lambda}\left[\frac{2 r^{3}-3 r_{2} r^{2}+\left(2 r_{1} r_{2}-r_{1}^{2}\right) r}{\left(1-r_{1} / r_{2}\right)^{2}}\right]- \\
& \frac{5 N \rho \pi^{2} \mu^{2} \omega^{2}\left(r_{2}^{4}-r_{1}^{4}\right)}{9 m_{v} R^{2} \dot{m} \lambda}\left[\frac{2 r_{2} r^{2}-r^{3}-\left(2 r_{1} r_{2}-r_{1}^{2}\right) r}{\left(1-r_{1} / r_{2}\right)^{2}}\right]
\end{aligned}
$$

两边对变量 $r$ 积分并考虑边界条件 $\left.\Theta_{w}\right|_{r=r_{1}}=0$, 得

$$
\begin{aligned}
\Theta_{w}= & \frac{\pi \mu \omega^{2}}{2 c \dot{m} h}\left(r^{4}-r_{1}^{4}\right)+\left(\frac{\mu \omega^{2}}{12 \lambda}+\frac{\pi \rho^{2} h^{3} \omega^{4} r^{2}}{1680 \dot{m} \lambda}\right)\left[\frac{\left(r_{2}-r_{1}\right)^{2}-\left(r_{2}-r\right)^{2}}{\left(1-r_{1} / r_{2}\right)^{2}}\right]+ \\
& \frac{5 N \rho \pi^{2} \mu^{2} \omega^{2}\left(r_{2}^{4}-r_{1}^{4}\right)}{108 m_{v} R^{2} \dot{m} \lambda}\left[\frac{3 r^{4}-8 r_{2} r^{3}+6\left(2 r_{1} r_{2}-r_{1}^{2}\right) r^{2}+3 r_{1}^{4}-4 r_{1}^{3} r_{2}}{\left(1-r_{1} / r_{2}\right)^{2}}\right]
\end{aligned}
$$

即对偶盘表面温升 $\Theta_{w}$ 沿径向的解析表达式。

将式(23)代入方程式(18), 就得到 ATF 同时沿 径向和轴向变化的温度场理论表达式

$\Theta=\frac{\pi \mu \omega^{2}}{2 c \dot{m} h}\left(r^{4}-r_{1}^{4}\right)+$

$\left[\frac{\mu \omega^{2}}{\lambda}\left(\frac{z^{3}}{3 h^{3}}-\frac{z^{2}}{2 h^{2}}+\frac{1}{12}\right)+\frac{\pi \rho^{2} h^{3} \omega^{4} r^{2}}{1680 \dot{m} \lambda}\right]\left[\frac{\left(r_{2}-r_{1}\right)^{2}-\left(r_{2}-r\right)^{2}}{\left(1-r_{1} / r_{2}\right)^{2}}\right]+$

$\frac{5 N \rho \pi^{2} \mu^{2} \omega^{2}\left(r_{2}^{4}-r_{1}^{4}\right)}{108 m_{V} R^{2} \dot{m} \lambda}\left[\frac{3 r^{4}-8 r_{2} r^{3}+6\left(2 r_{1} r_{2}-r_{1}^{2}\right) r^{2}+3 r_{1}^{4}-4 r_{1}^{3} r_{2}}{\left(1-r_{1} / r_{2}\right)^{2}}\right]$

由式(24)可见, 在初始制动的液体摩擦阶段, ATF 非稳态传热过程中的温升 $\Theta$ 关于轴向坐标 $z$ 成立 方变化, 并与径向坐标 $r$ 的四次方、摩擦盘转速 $\omega$ 的四次方、油膜厚度 $h$ 的立方密切有关，而 $\omega$ 和 $h$ 都是与制动时间 $t$ 密切相关的。将式(24)代 回式(14), 即得到 ATF 的三维热流密度场的理论 分布。

特殊地, 当 $\mathrm{d} \omega / \mathrm{d} t=0$ 时, 式(24)最后一项变为 零, 并退化为非制动时带排扭矩作用条件下 ${ }^{[24]}$, 因液体黏性摩擦产生的 ATF 温度场的二维分布表 达式

$$
\begin{aligned}
\Theta= & \frac{\pi \mu \omega^{2}}{2 c \dot{m} h}\left(r^{4}-r_{1}^{4}\right)+\left[\frac{\left(r_{2}-r_{1}\right)^{2}-\left(r_{2}-r\right)^{2}}{\left(1-r_{1} / r_{2}\right)^{2}}\right]\left[\frac{\pi \rho^{2} h^{3} \omega^{4} r^{2}}{1680 \dot{m} \lambda}+\right. \\
& \left.\frac{\mu \omega^{2}}{\lambda}\left(\frac{z^{3}}{3 h^{3}}-\frac{z^{2}}{2 h^{2}}+\frac{1}{12}\right)\right]
\end{aligned}
$$

对仅有带排扭矩作用的非制动工况的热对流特 性研究，将另具文报道，不赘述。

\section{5 结果讨论}

\section{1 与以往试验结果的比较}

湿式制动器封闭腔内的温度难以测定, 获取可 靠的试验数据较为困难, 这里采用 HUANG 等 ${ }^{[7]}$ 液体 黏性传动在对偶盘转速为零时 $\left(\omega_{1}=0\right)$ 的试验测量数 据, 并与取得的试验结果加以比较。图 6 给出了本文 计算的压力 $p$ 解析解(式 9)与 HUANG 等 ${ }^{[7]}$ 的试验结 果对比情况, 结果表明二者符合得非常好, 最大相 对误差 $7.6 \%$ 。图 7 给出了本文计算的温升 $\Theta$ 解析解 (式(21)) 与 HUANG 等 ${ }^{[7]}$ 的试验结果对比, 发现二者 最大差异不超过 $2.8 \mathrm{~K}$, 解析模型的预测结果工程上 可接受, 证明了理论模型的正确性。

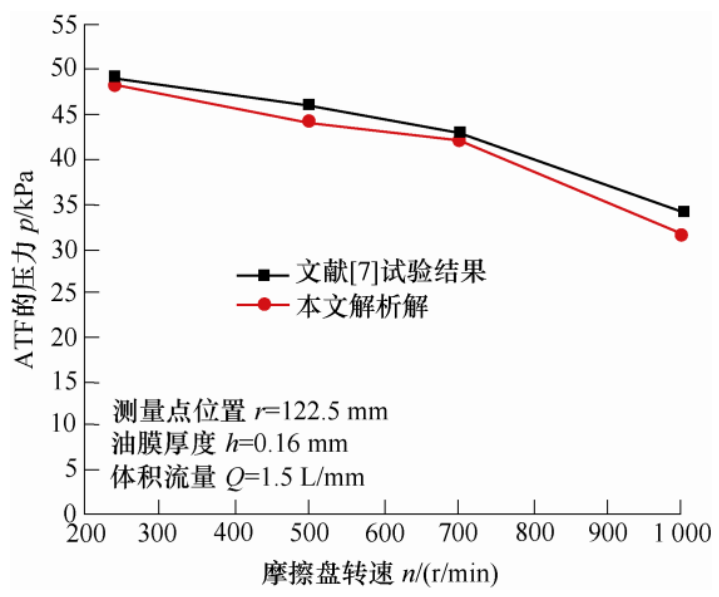

图 $6 \mathrm{ATF}$ 压力解析解与以往试验结果的对比

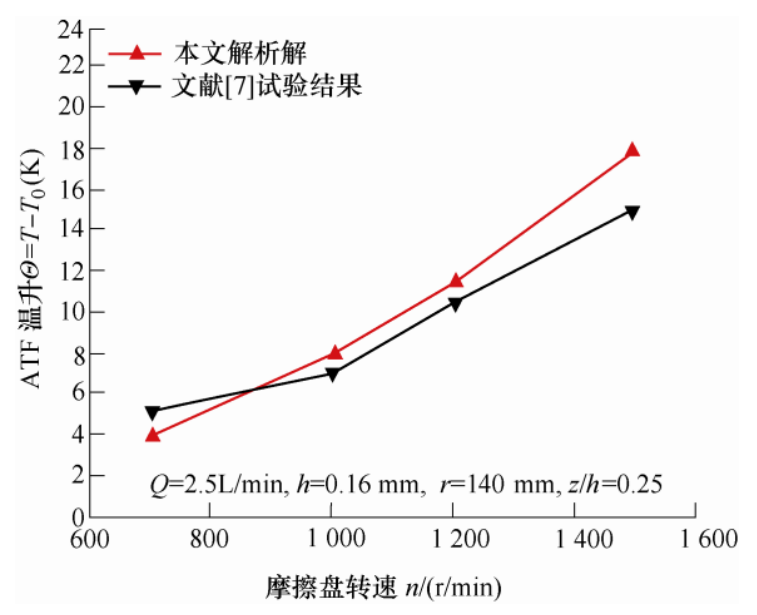

图 $7 \mathrm{ATF}$ 温度解析解与以往试验结果的对比 


\subsection{ATF 速度场的理论分布结果}

理论模型证明正确后, 下面用本文模型进行湿 式制动器热对流特性和参数分析。已知某型号双桥 矿用汽车, 满载总质量 $m_{V}=55 \mathrm{t}$, 在水平地面制动 时初速度为 $u_{0}=30 \mathrm{~km} / \mathrm{h}$, 车轮滚动半径 $R=0.8 \mathrm{~m}$, 前、后桥各配备 2 个湿式制动器, 设前、后制动器 制动力分配比例为 $2: 3$, 每个后桥湿式制动器的摩 擦副数 $N=20$ 。根据 ISO3450 和 GB/T21152 矿车制 动效能标准, 在制动器起作用时间 $t_{2}=0.05 \mathrm{~s}$ 时, 矿 车最大制动减速度大小 $a_{b m a x}=1.7 \mathrm{~m} / \mathrm{s}^{2}$, 如图 3 所示, 矿车湿式制动器其他参数见表 $1^{[13]}$ 。

\section{表 1 某型矿车湿式制动器参数}

\begin{tabular}{lc}
\hline \multicolumn{1}{c}{ 计算参数 } & 数值 \\
\hline 摩擦盘内径 $r_{1} / \mathrm{mm}$ & 210 \\
摩擦盘外径 $r_{2} / \mathrm{mm}$ & 292.5 \\
油膜初始厚度 $h_{0} / \mathrm{mm}$ & 0.016 \\
ATF 密度 $\rho /\left(\mathrm{kg} \cdot \mathrm{m}^{-3}\right)$ & 840 \\
ATF 动力粘度 $\mu / \mathrm{Pa} \cdot \mathrm{s}$ & 0.017 \\
ATF 热传导率 $\lambda / \mathrm{W} \cdot(\mathrm{m} \cdot \mathrm{K})^{-1}$ & 0.137 \\
ATF 定压比热容 $c / \mathrm{J} \cdot(\mathrm{kg} \cdot \mathrm{K})^{-1}$ & 2219 \\
ATF 入口温度 $T_{0} / \mathrm{K}$ & 293 \\
\hline
\end{tabular}

图 8 和 9 分别给出了 ATF 径向速度 $v_{r}$ 沿轴向和 径向的解析分布。由图 8 可见, $v_{r}$ 沿轴向呈抛物性 形对称分布, 且随着制动时间增加而增大, 这是因 为根据式(10), 矿车制动器在液体摩擦阶段转速相 对很低, 使得第二项离心力偏移效应很小, 径向速 度分布几乎左右完全对称, 就像 Poiseuille 流动那 样; 既然第一项占主导地位, 制动时间 $t$ 越长, 在 活塞压缩作用下油膜厚度 $h$ 越小, 故而在半径 $r$ 保 持不变时 $v_{r}$ 越大。注意到径向速度在这两个壁面处 大小为零, 这是牛顿流体的无滑移假设所致。由图 9 可见, 半径 $r$ 越大 $v_{r}$ 越小, 这是由于在流量一定 的条件下流动截面积增大所致; 制动时间 $t$ 越长, $v_{r}$ 越大, 这是因为当方程式(10)第一项占统治地位时, 在油膜相对厚度 $(z / h)$ 不变时, 制动压力作用下油膜 绝对厚度 $h$ 变薄的必然结果。

\subsection{ATF 温度场的理论分布结果}

图 10 给出了 ATF 与对偶盘壁面接触处的温升 分布。由 $\Theta_{w}$ 的解析表达式(19)可知, 虽然它与 $r^{4}$ 相关, 但最后总趋势为沿径向近似线性分布, 这与 以往许多数值仿真的研究结论相一致 ${ }^{[8,13,21]}$; 由图 10 还可看出, 制动时间 $t$ 越长 $\Theta_{w}$ 越大, 在指定参 数不变时, 式(19)中第一项占主导, 而该项和油膜 厚度 $h$ 成反比, 显然随着制动时间的增长油膜厚度

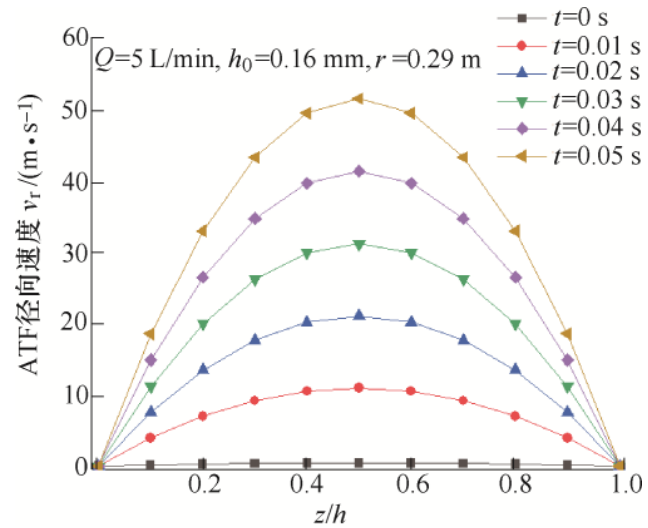

图 $8 v_{r}$ 沿轴向的理论分布 $(r=0.29 \mathrm{~m})$

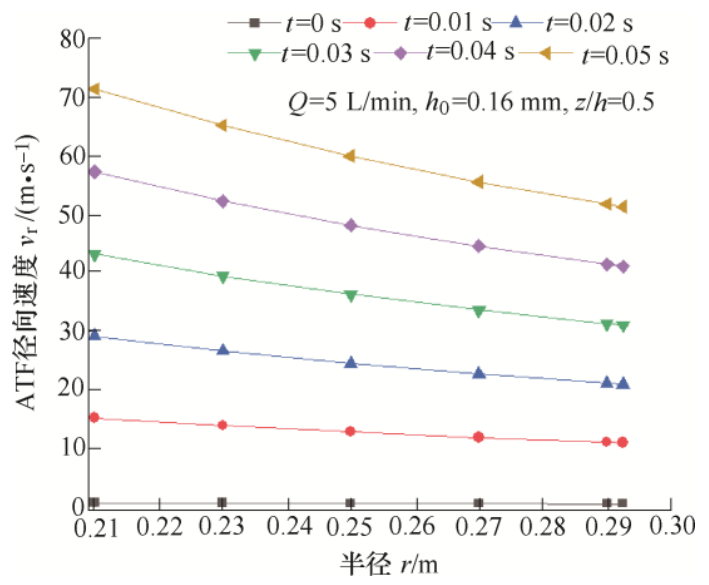

图 $9 v_{r}$ 沿径向的理论分布 $(z / h=0.5)$

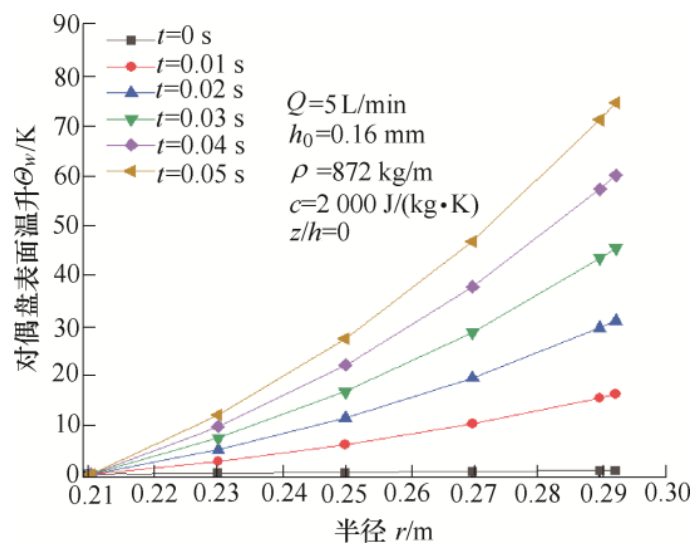

图 10 对偶盘表面温升沿径向的理论分布

越来越小。流体温升沿轴向分布的理论解由图 11 给 出。由图可见，在液体摩擦阶段，由于制动时间非 常短暂, 制动转速变化相对较小, 导致沿轴向的温 升几乎不变, 这与未制动时带排转矩作用下的温升 沿轴向变化趋势明显不同; 但是，由于瞬间产生的 热量非常多, 导致制动时间越长, ATF 温升越剧烈。 图 12 给出 ATF 在摩擦盘和对偶盘中间平面 $(z / h=0.5)$ 位置处的温升, 同时沿制动时间和径向的三维温度 分布理论解。由图可见, 显然内径处温升为零, 外 径处温升最大; 制动时间愈长, 温升越大。 


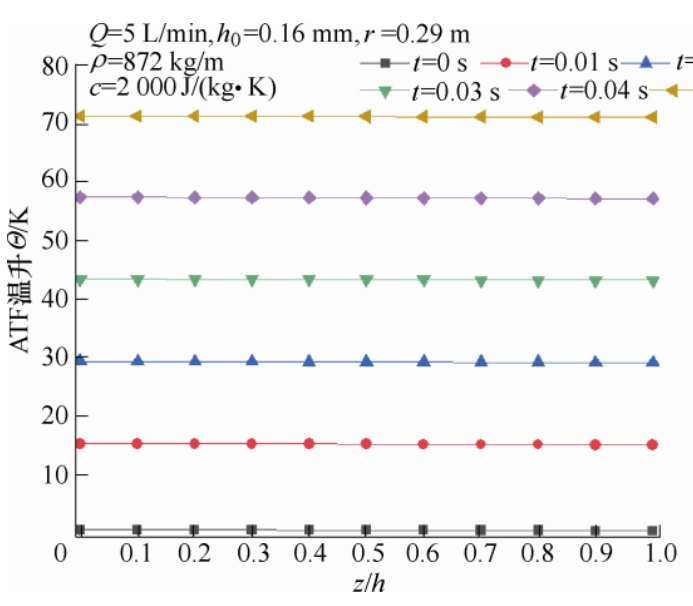

图 $11 \mathrm{ATF}$ 温升沿轴向的理论分布 $(r=0.29 \mathrm{~m})$

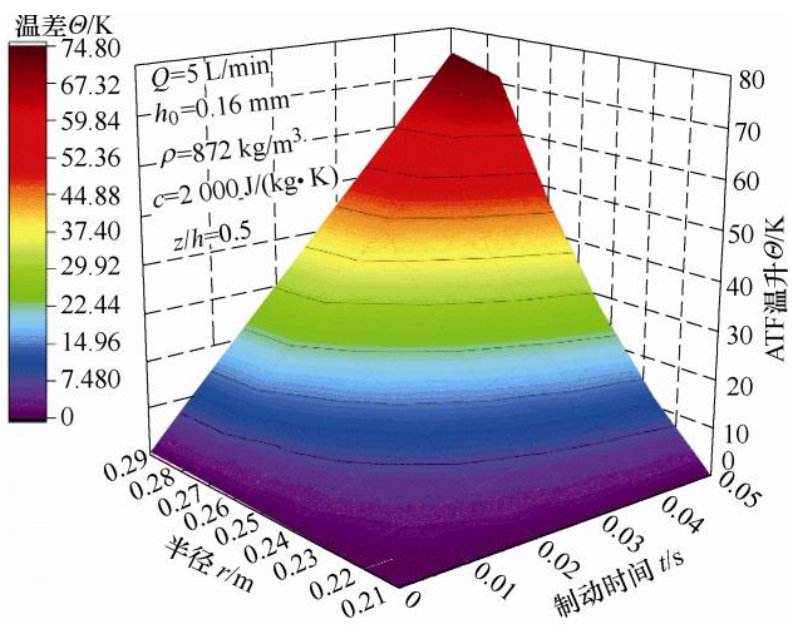

图 $12 \mathrm{ATF}$ 温升的三维理论分布 $(z / h=0.5)$

\subsection{ATF 热流密度场的理论分布结果}

随着制动时间的变大, 图 13、14 分别给出 ATF 轴向热流密度 $q_{z}$ 沿轴向和径向的三维理论分布。根 据 Soo 的边界层假设 ${ }^{[22]}$, 这里只考虑了轴向热传导 而忽略了轴向速度 $v_{z}$, 因而热对流无贡献。由这两 个图可见, 制动时间变大时轴向热流密度也相应增 大； $q_{z}$ 沿摩擦盘和对偶盘中间的平面对称分布，任 意制动时刻都在中间平面处达到极大值, 在圆盘外 径处达到最大值。注意在油液入口处、摩擦盘和对 偶盘表面的轴向热流密度均为零, 这是由第一类和 第二类边界条件决定的。

当制动时间变化时, ATF 径向热流密度 $q_{r}$ 沿轴 向和径向的三维理论分布分别如图 15、16 所示。由 数量级分析可知, 与对流大小相比, 径向热传导可 忽略, 故只考虑 ATF 径向存在因流体运动携带的温 差 $\left(T-T_{0}\right)$ 导致的对流贡献, 由热对流方程式(13)控 制。同样地, 在油液入口处、摩擦盘和对偶盘表面 的轴向热流密度均为零, 这是由第一类和第二类边 界条件的规定所致。在摩擦副外径处径向热流密度 $q_{r}$ 达到极大值, 具体在接近油膜厚度中间平面附近 的外径位置达到最大值。由图 13 16 对比可见, $q_{r}$
和 $q_{z}$ 的 3D 分布形状相似, 但是, 注意到由于流体 体积热容项 $\rho c$ 的放大作用, 导致 $q_{r}$ 数值远大于 $q_{z}$, 这是由热对流流体宏观运动的固有传热属性决定 的，此外二者的矢量方向也各不相同。

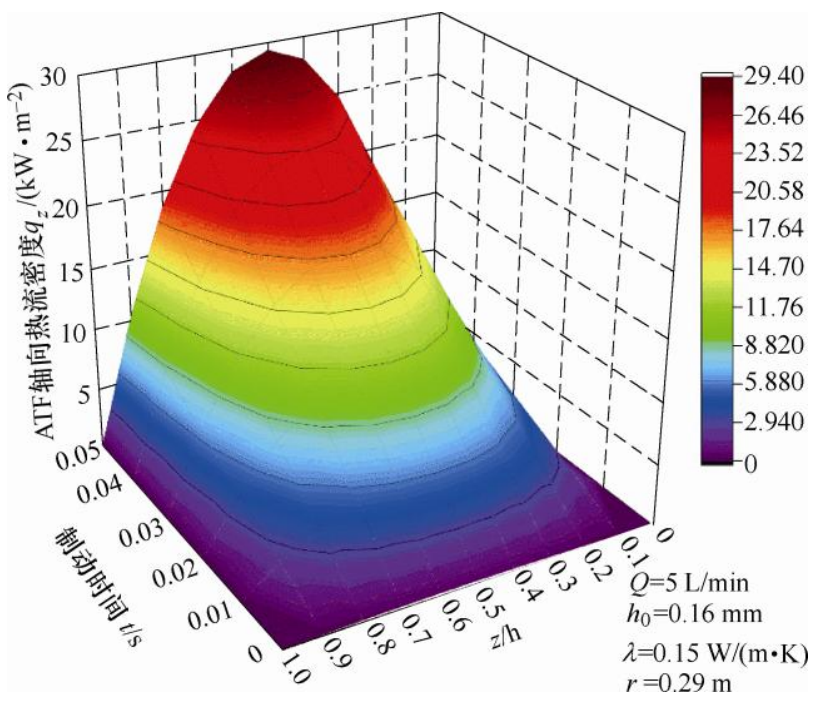

图 $13 q_{z}$ 随 $t$ 和 $z / h$ 的三维理论分布 $(r=0.29 \mathrm{~m})$

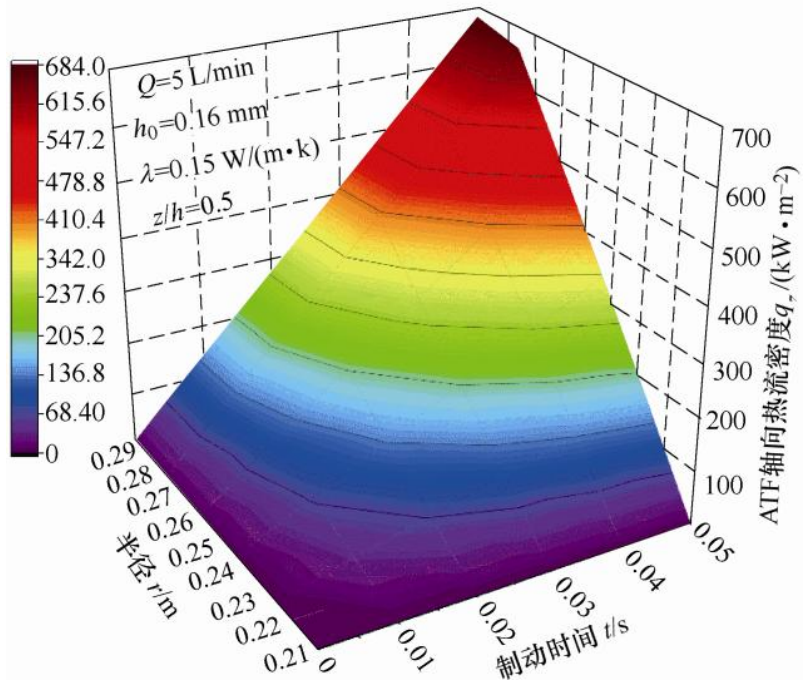

图 $14 q_{z}$ 随 $t$ 和 $r$ 的三维理论分布 $(z / h=0.5)$

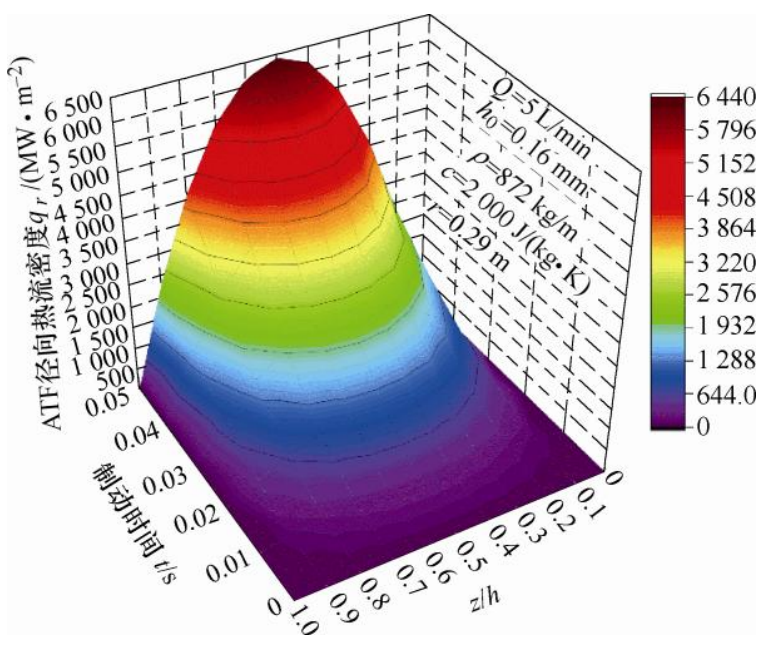

图 $15 q_{r}$ 随 $t$ 和 $z / h$ 的三维理论分布 $(r=0.29 \mathrm{~m})$ 


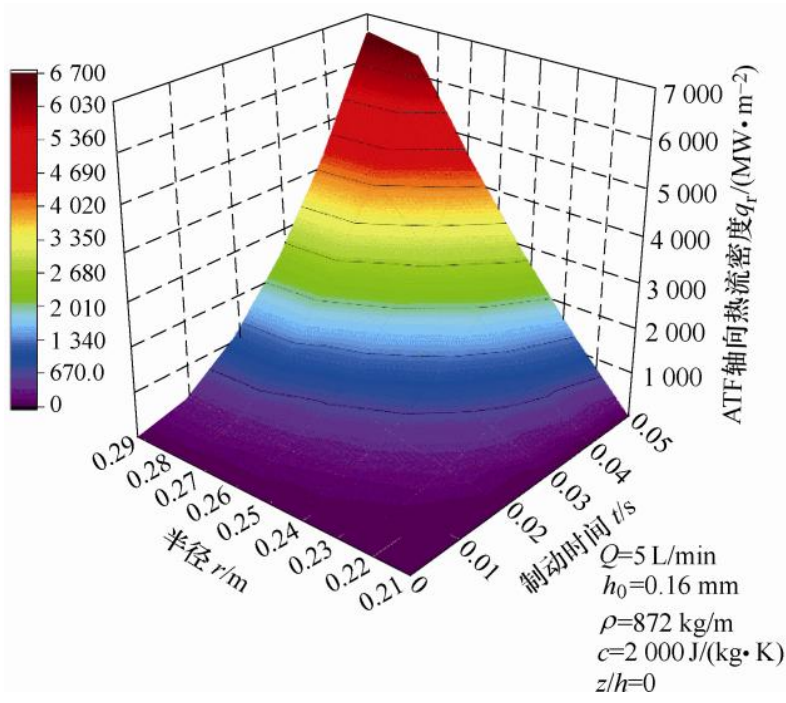

图 $16 q_{r}$ 随 $t$ 和 $r$ 的三维理论分布 $(z / h=0.5)$

\section{6 结论}

（1）考虑了湿式制动器初始制动阶段冷却流体 的黏性传动特征, 由简化的纳维-斯托克斯方程和多 项式分布假设, 同时考虑压力梯度和离心力影响, 建立了湿式制动器 $\mathrm{ATF}$ 的径向流动速度和压力的理 论模型, 获得了 ATF 速度场和流动压力的显式解析 表达式。

(2) 在液体摩擦制动阶段, 假设摩擦盘和对偶 盘为热绝缘体, 热量均由 ATF 带走, 建立了摩擦副 流体的非稳态能量积分方程, 采用了能够满足所有 径向和轴向边界条件的温度多项式分布形式, 获得 了能同时描述沿径向和轴向变化的流体温度解析表 达式, 以此为基础, 得到了初始制动阶段湿式制动 器 ATF 沿轴向和径向热流密度的三维理论分布, 通 过与以往试验结果对比证明了本文建立的压力和温 度理论解的正确性。

(3) 本文建立的湿式制动器 ATF 非稳态能量积 分方法，以及同时考虑沿轴向、径向多项式形式的 温度分布, 有潜力推广应用到液黏离合器、液黏测 功机等其他 HVD 装置中去。

\section{参 考 文 献}

[1] 赵波. 交流传动电动轮自卸车结构与设计 $[\mathrm{M}]$. 北京: 中国铁道出版社, 2013.

ZHAO Bo. Structures and design for off-highway motorized wheel dump trucks with AC electric drive system[M]. Beijing: China Railway Publishing House, 2013.

[2] 赵波, 陈东升. 液粘测功机的带排扭矩理论与试验研
究[J]. 兵工学报, 1997(2): 22-28.

ZHAO Bo, CHEN Dongsheng. The theoretical and experimental study on the drag torque of a hydroviscous dynamometer[J]. ACTA Armamentarii, 1997(2): 22-28.

[3] WANG Yanzhong, WEI Bin, Wet multi-disc friction components heat dissipation capability and optimal oil supply under continuous braking condition[J]. Industrial Lubrication and Tribology, 2014, 66(6): 653-661.

[4] ADAMOVICZ A, GRZES P. Influence of convective cooling on a disc brake temperature distribution during repetitive braking[J]. App. Therm. Eng., 2011, 31: 2177-2185

[5] MICHAEL R A. Key elements of wet brake and clutch design[C/CD]//SAE Technical Paper Series No. 921660, International Off-highway \& Powerplant Congress \& Exposition, Milwaukee, Wisconsin, Sep 14-17, 1992.

[6] QING Datong, SUN Dongye. Research on design method of a multiple disc wet brake in lubricated environment[J]. Chin. J. Mech. Eng., 2003(4): 391-395.

[7] HUANG Jiahai, WEI Jianhua, QIU Minxiu. Laminar flow in the gap between two rotating parallel frictional plates in hydro-viscous drive[J]. Chin. J. Mech. Eng., 2012, 25(1): 144-152.

[8] XIE Fangwei, CUI Jianzhong, SHENG Gang, et al. Thermal behavior of multidisk friction pairs in hydroviscous drive considering inertia item[J]. ASME J. Tribol., 2014, 136(4): 041707.

[9] SINGH $\mathrm{S} \mathrm{K}$, ABBASSI $\mathrm{H}$, TAMAMIDIS P. 3D investigation into the thermal behavior of the wet multi-disk axle brake of an off-highway machinery[J]. App. Therm. Eng., 2018, 136: 576-588.

[10] JEN T, NEMECEK D J. Thermal analysis of a wet-disk clutch subjected to a constant energy engagement[J]. Int. J. Heat Mass Transf., 2008, 51: 1757-1769.

[11] ABDULLAH O I, SCHLATTMANN J. An investigation into the thermal behavior of the grooved dry friction clutch[J]. ASME J. Tribol., 2014， 136(3): 034504.

[12] JANG J Y, KHONSARI M M. On the formation of hot spots in wet clutch systems[J]. ASME J. Tribol., 2002, 124(2): 336-345.

[13] SERGIENKO V, TSELUEV M, KOLESNIKOV V I, et al. Prediction of thermal conditions for multidisc oil-cooled brake for a mining truck[R]. SAE Technical Paper Series, 2010-01-1713.

[14] BELHOCINE A, OMAR W Z W. Computational fluid 
dynamics (CFD) analysis and numerical aerodynamic investigations of automotive disc brake rotor[J]. Australian Journal of Mechanical Engineering, 2018, 16(3): 188-205.

[15] GRZES P. Determination of the maximum temperature at single braking from the FE solution of heat dynamics of friction and wear system of equations[J]. Numerical Heat Transfer, Part A: Applications, 2017, 71(7): 737-753.

[16] YEVTUSHENKO A A, GRZES P. Mutual influence of the sliding velocity and temperature in frictional heating of the thermally nonlinear disc brake[J]. Int. J. Therm. Sci., 2016, 102: 254-262.

[17] 赵波, 王梓羽, 惠源. 考虑液体黏性传动的湿盘制动器 ATF 温度场的解析模型[J]. 机械, 2019, 46(12): 1-11. ZHAO Bo, WANG Ziyu, HUI Yuan. 2D analytical solutions of thermal behavior for automotive transmission fluid considering hydroviscous drive in wet brakes[J]. Machinery, 2019, 46(12): 1-11.

[18] APHALE C R, SCHUlTZ W W, CECCIO S L. The Influence of grooves on the fully wetted and aerated flow between open clutch plates[J]. ASME J. Tribol., 2010, 132(1): 011104.
[19] IQBAL S, AL-BENDER F, BERT P, et al. Model for predicting drag torque in open multi-disks wet clutches[J]. ASME J. Fluids Eng., 2014， 136(2): 021103.

[20] NEUPERT T, BENKE E, BARTEL D. Parameter study on the influence of a radial groove design on the drag torque of wet clutch discs in comparison with analytical models[J]. Tribol. Int., 2018, 119: 809-821.

[21] JANG J Y, KHONSARI M M. Thermal characteristics of a wet clutch[J]. ASME J. Tribol., 1999, 121(3): 610-617.

[22] SOO S L. Laminar flow over an enclosed rotating $\operatorname{disk}[\mathrm{J}]$. Trans. ASME, 1958, 80: 287-296.

[23] ELLWOOD C M, KORCHINSKY W J. The heating, by viscous dissipation, of liquids flowing across an enclosed rotating disc[J]. Int. J. Heat Mass Transf., 2000, 43: 1035-1050.

[24] YUAN Shihua, PENG Zengxiong, JING Chongbo. Experimental research and mathematical model of drag torque in single-plate wet clutch[J]. Chin. J. Mech. Eng., 2011, 24(1): 91-97.

作者简介: 赵波, 男, 1972 年出生, 博士, 教授。主要研究方向为车辆 湿盘式制动器性能分析和传热传质统一场理论。

E-mail: zhaobo@scu.edu.cn 